LA W RENCE LIVERMORE NATIO NAL LABORATORY
LLNL SMP Light Diffuser
Fabrication and Preliminary Data

W. Small IV

June 5, 2006 
This document was prepared as an account of work sponsored by an agency of the United States Government. Neither the United States Government nor the University of California nor any of their employees, makes any warranty, express or implied, or assumes any legal liability or responsibility for the accuracy, completeness, or usefulness of any information, apparatus, product, or process disclosed, or represents that its use would not infringe privately owned rights. Reference herein to any specific commercial product, process, or service by trade name, trademark, manufacturer, or otherwise, does not necessarily constitute or imply its endorsement, recommendation, or favoring by the United States Government or the University of California. The views and opinions of authors expressed herein do not necessarily state or reflect those of the United States Government or the University of California, and shall not be used for advertising or product endorsement purposes.

This work was performed under the auspices of the U.S. Department of Energy by University of California, Lawrence Livermore National Laboratory under Contract W-7405-Eng-48. 


\section{LLNL SMP Light Diffuser Fabrication and Preliminary Data}

We are developing a cylindrical light diffuser using shape memory polymer (SMP) whose diameter, length, stiffness, and diffusion profile can be tailored to suit a particular application.

The cylindrical SMP diffuser is made by casting SMP around the end of a glass optical fiber using a teflon tube as the casting mold, and abrading the cured SMP surface to cause the light to leak radially outward. The inner diameter of the casting tube is slightly larger than the fiber diameter. A smaller teflon tube is positioned over the fiber (between the fiber and the casting tube) to approximately center the fiber tip in the casting tube. As the SMP cures, it bonds with the optical fiber, creating a strong joint without the need for additional adhesives or mechanical fixtures. A close-up of the SMP-fiber joint and the finished SMP diffuser are shown in Fig.1. The SMP formulation (developed in-house) was specifically designed to be optically transparent in the visible and near-infrared regions; the spectral absorption of the SMP is shown in Fig. 2. The low absorption is important because (1) it allows the light to travel the length of the diffuser without suffering excessive loss due to absorption and (2) it permits delivery of up to $7 \mathrm{~W}(300 \mu \mathrm{m}$ SMP rod on $100 \mu \mathrm{m}$ core multimode fiber) of laser power into the diffuser without damaging the diffuser. SMP is a good wave guiding material with a refractive index of approximately 1.5. Also, the SMP stiffness can be tailored from stiff (e.g. acrylic, Ea $10^{9} \mathrm{~Pa}$ ) to very flexible (e.g. silicon rubber, Ea $\sim 10^{6} \mathrm{~Pa}$ ). Finally, since SMP can self-actuate, the SMP diffuser could be designed to actuate into a shape other than a straight rod (e.g. 2D or 3D coil).

A computer-controlled abrasive media-blasting machine was built to create the diffusing SMP surface. The blasting pen is mounted on a vertical translation stage and the SMP is fixed on a rotation stage, both controlled using LabVIEW software. Parameters such as pen tracking speed, sample rotation speed, blasting pressure, abrasive media material, media feed rate, and blasting distance can be controlled to achieve the desired diffusing characteristics. We have aimed to make a diffuser that has uniform $360^{\circ}$ radial light emission over a length of 2-3 $\mathrm{cm}$. Should the need arise, other more complex or specialized diffusing patterns are possible using the media blasting

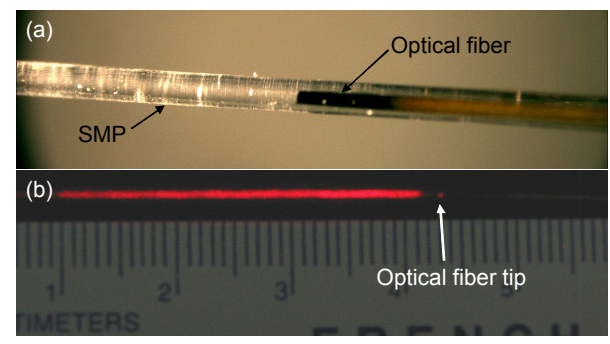

Fig. 1. (a) Joint formed by casting SMP over the optical fiber tip. The blackened area of the optical fiber was caused by burning off the protective polyimide coating in order cleave the fiber tip prior to casting. (b) SMP diffuser coupled to a red diode laser (diffusion length $=3 \mathrm{~cm}$ ). SMP diameter $=0.300 \mathrm{~mm}$. Optical fiber diameter $=0.125 \mathrm{~mm}$.

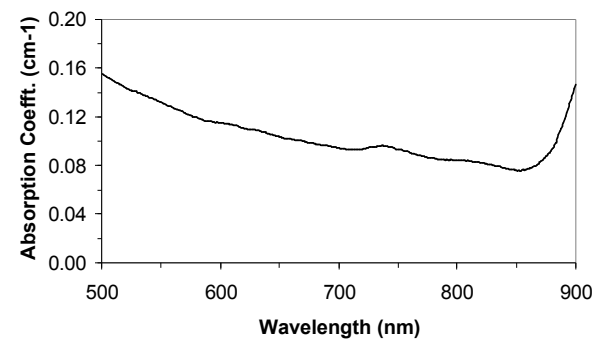

Fig. 2. Absorption coefficient of the SMP as a function of wavelength (raw absorbance data acquired using a Cary 300 spectrophotometer). system. We have made SMP diffusers of $0.3-1.1 \mathrm{~mm}$ diameter which emit $\sim 60 \%$ of the coupled light in a radial direction over lengths of $3 \mathrm{~cm}$ (Fig. 1). As described below, further optimization of the diffuser performance is underway.

A testing protocol has been developed to quantify the diffusion performance for different blasting parameters and optimize the diffuser fabrication process. To measure the light diffusion profile along the length of the diffuser, a low power diode laser $(4 \mathrm{~mW}, 670 \mathrm{~nm})$ is coupled to the diffuser and a 12-bit image of the emitting diffuser is acquired using a Photometrics CoolSnap HQ camera. Matlab software is used to obtain the average pixel intensity across the width of the diffuser, which is plotted as a function of axial distance along the diffuser length ("diffusion profile"). The amount of light emitted from the diffuser is measured using an integrating sphere to quantify the diffusion efficiency (percentage of the coupled light emitted radially). The diffusion performance for several abrasive media materials is shown in Fig. 3. Sodium bicarbonate particles yield a more uniform axial diffusion profile compared to 50-150 micron aluminum oxide particles at the expense of reduced diffusion efficiency for the given blasting 
parameters. As is evident in the data, there is a trade-off between axial uniformity of the diffusion profile and diffusion efficiency. Axial diffusion profiles for various blasting pressures and blasting distances are

(a)

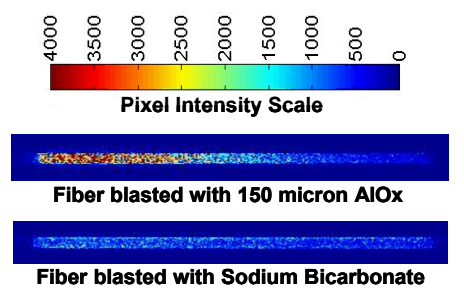

(b)

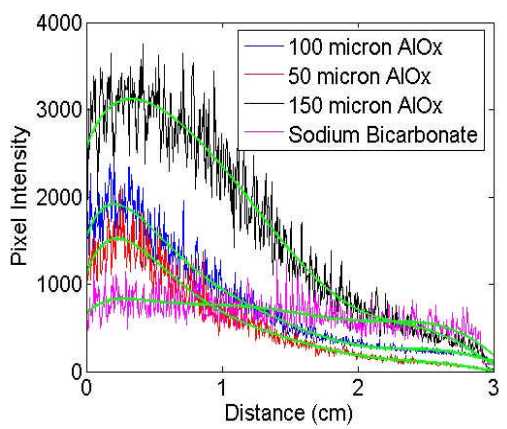

(c)

\begin{tabular}{|c|c|}
\hline Abrasive Media & $\begin{array}{c}\text { Light Diffusion } \\
\text { (\%) }\end{array}$ \\
\hline 50 micron AlOx & 90 \\
\hline 100 micron AlOx & 90 \\
\hline 150 micron AlOx & 85 \\
\hline Sodium Bicarbonate & 59 \\
\hline
\end{tabular}

Fig. 3. Light emittance of SMP diffusers made using different abrasive media: aluminum oxide and sodium bicarbonate particles. (a) Intensity image of diffusers. (b) Average intensity across the diffuser width plotted as a function of axial distance along the diffuser ("diffusion profile"). (c) Diffusion efficiency for each material given as the percent of total coupled light emitted radially. Blasting parameters: 30 PSI blasting pressure, $7 \mathrm{~cm}$ blasting distance, $2 \mathrm{~mm} / \mathrm{s}$ tracking speed.

(a)

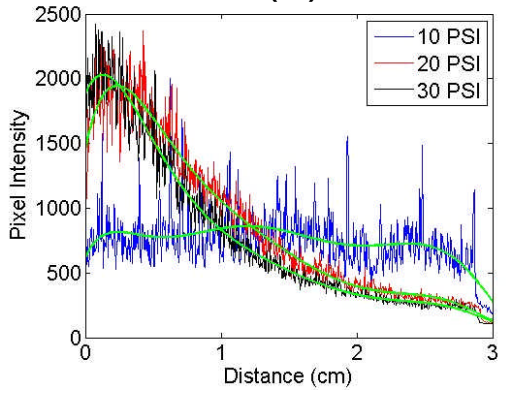

(b)

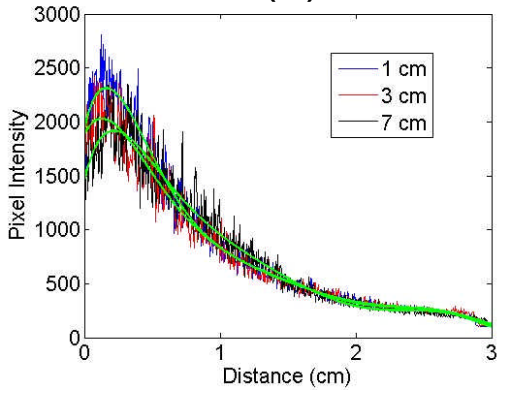

(c)

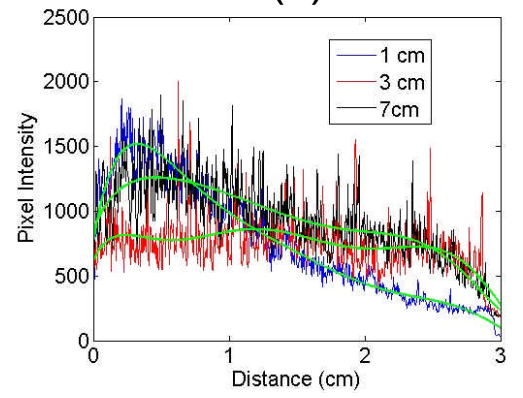

Fig. 4. Diffusion profiles for different blasting pressures and blasting distances. Blasting parameters: 100-micron aluminum oxide, $2 \mathrm{~mm} / \mathrm{s}$ tracking speed, (a) $3 \mathrm{~cm}$ blasting distance, (b) 30 PSI and (c) 10 PSI blasting pressures.

shown in Fig. 4. For given blasting parameters, the diffusion profile becomes more uniform only when the blasting pressure is reduced to 10 PSI. Further, the blasting distance has little impact on diffusion characteristics until the blasting pressure is reduced to 10 PSI. There is almost no impact on the diffusion profile for blasting distances from 1 to $7 \mathrm{~cm}$ at 30 PSI. However, at 10 PSI the blasting distance appears to have a slight effect, with the $3 \mathrm{~cm}$ and $7 \mathrm{~cm}$ blasting distances creating a flatter diffusion profile.

Initial experiments indicate that relatively low blasting pressures, between 10 and 30 PSI, and the sodium bicarbonate abrasive material yield the best diffusers. Further optimization of the axial diffusion uniformity and efficiency may be achieved by gradually increasing the degree of surface abrasion along the length of the diffuser, allowing more light to be emitted further along the diffuser length. Preliminary work has demonstrated that such a "roughness gradient," accomplished by gradually reducing the tracking speed of the blasting pen, can improve the diffuser performance. 